\title{
Implementation of Cross Layer Design for Efficient Power and Routing in UAV Communication Networks
}

\author{
Haque NAWAZ ${ }^{1,2, *}$, Husnain MANSOOR ALI ${ }^{1}$ \\ ${ }^{1}$ Shaheed Zulfikar Ali Bhutto Institute of Science and Technology, Karachi, Sindh, Pakistan. \\ ${ }^{2}$ Sindh Madressatul Islam University, Karachi, Sindh, Pakistan. \\ hnlashari@smiu.edu.pk (*Corresponding author)
}

\begin{abstract}
The unmanned aerial vehicle communication networks (UAVCN) is an emerging technology of wireless communication. By making use of this technology, the swarm of unmanned aerial vehicles (UAVs) forms a network in which the UAVs can communicate with each other and trigger the information for a particular operation of military and civilian applications. The UAV nodes frequently face design issues and power limitations' which affect the routing mechanism. It is a unique challenge for the researchers to introduce the efficient power and routing mechanism that can improve the performance of UAVs' communication networks. However, the concept of cross layer design and efficient power algorithm proposed in this paper increases the performance of UAVCN. The proposed approach integrates the layers 1,2,3 (physical, link, and network). By implementing this kind of approach, an efficient power optimized link state routing (EPOLSR) protocol introduced in this research modifies the conventional OLSR. The EPOLSR and OLSR are implemented and assessed in the first experiment scenario of UAVs communication networks by using an optimized network engineering tool. Moreover, the EPOLSR, OLSR, AODV, and DSR are implemented and assessed in the second experiment scenario. In these testbed experiments, it has been observed that EPOLSR performs better than other routing protocols for UAVs communication networks by increasing the throughput and minimizing the delay.
\end{abstract}

Keywords: UAVs, UAVCN, Cross layer design, Efficient power, Routing, EPOLSR.

\section{Introduction}

In wireless communication networks, an emerging technology is represented by UAVs' communication. It comes under the umbrella of a mobile adhoc network (MANET), which uses the infrastructure-less environment. The mobile nodes can share the information with each other during communication in the air. Mostly these UAVs are used for military (Dolicanin et al., 2018; Manyam et al., 2020) and civilian applications, generally in real-time reconnaissance and surveillance operations (Srinivasan et al., 2004; Bekmezci, 2013; Rajan et al., 2019; Singh et al., 2014).

Swarm of UAVs can communicate by utilizing the wireless LAN (WLAN) atmosphere. The nodes are facing power, mobility management, routing, service discovery, and design problems, that exists in the protocol stack layers (Shakhatreh et al., 2019). The architecture of layers is the design of protocols that helps in interlayer communication. The standard interfaces are available in the reference layered architecture, which supports interlayer interaction (Li, Jia \& Du, 2006).

Each layer constraint is addressed in the link layer coding, power control, adaptive techniques, medium access control (MAC) scheduling, and in the network layer routing addressed.
In UAVCN, an issue is to find the accurate, precise, and correct route to the destination and maintain the connectivity during the topology change, which breaks communication. In the different routing protocols, one or many metrics govern(s) the best routes. Still, the power is an issue that affects the transmission of crosslayer design, which disturbs the protocol stack from layer one to layer four, i.e., physical, link, network, and transport layer which distresses the metrics, e.g., delay, throughput, and energy consumption (Kawadia \& Kumar, 2005) thus, impacts the physical layer, determines the neighboring nodes that can hear the packet and, thus, the network layer affects interference which causes congestion and, thus, affects the transport layer. It is also key to several performance measures such as throughput, delay, and energy consumption. The challenge is to determine where in the architecture the power control problem is to be situated, to determine the appropriate power level by studying its impact on several performance issues, to provide a solution which deals properly with the multiple effects of transmit power control, and finally, to provide a software architecture for realizing the solution. We distill some basic principles on power control, which inform the subsequent design process. We then detail the design of a 
sequence of increasingly complex protocols, which address the multidimensional ramifications of the power control problem. Many of these protocols have been implemented, and may be the only implementations for power control in a real system. It is hoped that the approach in this paper may also be of use in other topical problems in cross-layer design. The cross-layer method is considered an effective mechanism to improve performance. It permits the constraints to be passed from the end-to-end layers. In addition, (Chen et al., 2007). IEEE stated that routing is the technique that chooses the best route by using the shortest hop metric. The energy efficient routing is a significant problem which can be addressed (Gupta et al., 2016; Cruz et al., 2018). However, the cross-layer technique can be considered in order to explore the performance significantly. Moreover, (Casaquite \& Hwang, 2006) discuss the scheduling power control to find out the best possible tx power, which sustains the signal-tointerference plus noise ratio (SINR) constraint, and, besides, the required data rate in ad-hoc wireless networks. However, it is inconvenient to solve the power control issue and maximize the lifetime of the network.

The researchers (Ghada et al., 2009) have introduced an approach to maintain and control routes in MANET and decrease the intra network interference and increase the lifetime of the network. The concept of integration among route selection and power control mechanisms has been introduced by assuming the node's selfish deportment, in which every node will vary its tx power despite the decision and status of other nodes. The cross-layer and modified adhoc on demand distance vector (AODV) routing protocol proposed in this research is carried out by simulation. The obtained results demonstrate that it increases the network lifetime, consumes less energy, and minimizes the delay. The authors (Zeng, 2019) discuss the UAV consumption of energy. The cross layer design and efficient power algorithm proposed in this paper was designed to increase the routing mechanism. This approach has been implemented. The EPOLSR has been introduced by modifying OLSR so that the routing performance of unmanned aerial vehicle communication networks can be improved.
The rest of this paper is structured as follows: Section 2 presents the literature review of the adapted routing protocols. Section 3 describes the cross layer design and section 4 proposes the employment of this cross layer design (CLD) for efficient power and routing. Section 5 presents the algorithm utilized for efficient power, while section 6 highlights the implementation of EPOLSR routing protocol. The first and the second experiments are demonstrated in sections 7 and 8, respectively. Finally, section 9 presents the conclusion of the paper.

\section{Literature Review}

The existing literature regarding to routing protocols has been studied and come up with the solution to implement the new idea that helps to solve the problem. The OLSR protocol has unique characteristics as compared to other protocols. The main feature of OLSR is its proactive nature (Wang et al., 2013). It keeps up an information table (Khan et al., 2019). Thus, it is called table driven. It is an algorithm that selects the best or identical route by discovering numerous features such as bandwidth, a load of the link, delay, etc.

The authors (Alshabtat \& Dong, 2011) proposed a new routing protocol for Unmanned Aerial Vehicles (UAVs have proposed an enhanced version of OLSR protocol that is known as a directional optimized link state routing (DOLSR) protocol. This protocol has the advantage of optimizing the end to end delay (E2E). The proposed protocol is better for real-time communication. This protocol hardware doesn't permit UAVs to operate with omnidirectional antennas. Also, proactive routing protocols exchange the topology control (TC) periodically, which consumes more power and bandwidth (BW) resources.

The researchers (Zheng et al., 2014) have introduced the mobility and load aware optimized link state routing protocol (ML-OLSR), which is an extension of OLSR. The enhancement of this protocol is carried out in three phases: the neighbor discovery, the MPR choosing, and the selection of an appropriate route. ML-OLSR uses the mobility aware algorithm and load-aware algorithm to choose the neighbor and route the traffic. In the

https://www.sic.ici.ro 
mobility-aware algorithm, the mobility-model uses the two novel routing metrics. One is the stability degree of a node (SDN), and the other is the reachability degree of a node (RDN). The authors (Rosati et al., 2013) have proposed an extension version of OLSR known as a predictiveoptimized link state routing protocol (P-OLSR). The characteristic of this routing protocol is the prediction of the quality of the link among unmanned aerial vehicles.

\section{Cross Layer Design}

The concept of cross layer design is a novel approach through which network resources can be utilized efficiently among the layers for data sharing. The authors (Amin et al., 2015) have proposed a cross-layer model that disseminates information in a peer-to-peer (P2P) network in ad-hoc mode. The issue raised by the authors refers to the fact that the routing overhead is a metric which impacts on redundant communication. However, the crosslayer technique exchanges the information by producing new interfaces among layer7 and layer 3. The exchange of data takes place in both directions among these layers and reduces the overhead. Additionally, the specific interfaces are introduced to support the operations independently. However, this is a different approach concerning the traditional layer. The Gnutella protocol shows an improved performance in terms of transmitting and receiving the data to lower layers. This protocol is used for peer to peer networks. It creates problems in peer discovery when implemented for an ad-hoc mode of the network environment. Flooding takes place for peer discovery based on the application layer, which increases the routing overhead. Therefore, the Gnutella protocol performs the function at application layer7 while AODV operates network layer3. The cross-layer Gnutella receives messages from nodes events at layer7. This technique minimizes the duplication of messages and maximizes the $p 2 p$ network performance in ad-hoc networking.

The authors (Bennis et al., 2017) have proposed the cross-layer technique for video transfer. In this study, the researchers have provided the concept of multipath forwarding protocol, which enables an efficient video transferring from the source to the base station. The application layer services are prioritized with high priority and low priority video frames. In the proposed scheme, the algorithm 1 is used to send packets to the routing layer and it also uses the MAC layer. According to the H.264 standard. I, P, and B frame are used. When the packet belongs to I-frame, then the construction path algorithm is used. However, when the packet belongs to the $\mathrm{P}$ and $\mathrm{B}$ frame, then the PB construction path algorithm is used. Otherwise, the CBR construction path algorithm is used. The source node chooses the next hop based on the I construction path algorithm for I frame packets. The intermediary node repeats this process until each packet reaches the destination. For this purpose, every chosen node is tagged as reserved I. This demonstrates that these are the only nodes that are responsible for I frame forwarding. After this, it triggers the information to its neighbors with its new current status and sends it to neighbors.

The authors (Shirani, R., St-Hilaire, M. \& Kunz, T. 2012) have proposed a cross-layer paradigm that integrates unmanned aeronautical ad-hoc networks (UAANETs) in two applications such as search and surveillance. This supports searching and tracking missions. The unified cross-layer is introduced based on the modular layering architecture, and its mechanism provides the interactions among cross-layer and strict layers. Ultimately this technique supports both applications. These capabilities of the proposed cross-layer architecture are similar to those of the traditional architecture of the UAVs. A network stack responsible for mutual communication with different layers is introduced. The stack module can ensure the functionality of crosslayer communication. The information received from the UAVs' navigation system or sensors is updated regularly. Similar data are used to offer services to different layers by using the network status stack. In this network stack module, different layers protocol information fields which revealed the concept of network status (Conti et al., 2004). Protocols utilize the information either from the top layer or the bottom layer to optimize the functionality. In the 
UAVs navigation system, the sensors receive the navigation information from the network status stack. However, the data gathered from other UAVs are served to different layers. This status information is regularly updated, and it ensures the contemporary network status.

The store carries forward based UAANET (SCFUAV) mechanism proposed in the architecture is implemented in the bundle layer. This concept is not available in traditional architecture. The delay tolerant data is handled by this layer (Jonson et al., 2008). The stabilized wireless links in a UAANET (SWUAV) mechanism proposed for the UAVs links stability are employed to reduce the delay, and the error-prone stable links are required during the UAVs random movement. However, the channel characterization research can be carried out to introduce the routing metric for suitable and durable links among UAVs.

The authors ( $\mathrm{Gu} \&$ Zhu, 2013) have discussed the cross-layer design and have explored the mobile ad-hoc network by combining the first three layers. The problem which is addressed is represented by the prediction of interference duration along the route, which is imposed by the neighbor nodes at each hop. Subsequently, the link breakage and the interference issue are highlighted. However, the authors have used the cross-layer design technique. They have proposed a minimum interference routing (MIR) protocol, which is considered an interference-aware routing mechanism, in which a new metric is introduced to guarantee that, at a low interference, the route links will not be disturbed.

The authors (Divya, Subasree \& Sakthivel, 2015) have proposed the approach known as minimal total transmission power routing (MTPR), which is the reactive approach to discover a path at minimum total tx power. By using the routing mechanism of MTPR, the path between source and destination is chosen. It computes the network efficiency according to the density of nodes having minimum residual battery power. Initially, this mechanism calculates all the routes of the total transmission power and selects an optimized way. MTPR performs the routes calculation of the route operation as:

$L=n_{0}, n_{1}, n_{2}, \ldots \ldots . ., n_{d}$
In route $\mathrm{L}$, the total transmission power is computed as:

$$
P_{t}=\sum_{i=1}^{d} P\left(n_{i}, n_{i+1}\right) \forall \operatorname{nodesn}_{i} \in L
$$

The node $\mathrm{k}$ power can be computed as:

$P_{k}=\min \left(P_{t}\right)$

However, during the data transmission, MTPR depends on the bit error ratio and the interference that is drawn back and produces a more significant E-2-E delay. The efficient power-aware routing (EPAR) is another reactive approach that proves to be more efficient when it escalates the lifetime of the network. It discovers all the nodes which are present in the network and identifies the total power. Besides, it determines the lost energy of a specific link and chooses an optimal route among all the neighbors by utilizing the minimax formulation. It also uses a traffic density factor to improve the packet delivery ratio (PDR). This mechanism minimizes the total variance in residual battery power (RBP) of every node through dynamic topology (DT).

By using the min-max formulation technique, the total power can be computed as:

$\max _{k}=(k) T_{k}(t)=\min _{i \in k}(k) T_{i}(t)$

The consumed power (CP) can be computed as:

$$
E_{c}=\sum_{i=1}^{k} T\left(n_{i}, n_{i+1}\right)
$$

The dense network due to the high movement of nodes increases the overload messages. The more significant topology changes produce more overhead. In this study, the authors discuss the performance metrics for the routing protocol efficiency concerning throughput, power consumption, residual energy, and network lifetime. The residual battery power metric is used for the performance evaluation of power aware routing as the power is the total amount of information routed by a node vs. the residual battery power of a considered node, which is computed by using the formula.

\section{ResidualBatteryPowerofknode}

$=\sum_{k}$ powerofknodeattimet 
The power consumption is a metric that is taken into consideration when the battery power is consumed because of the communication of data packets. If a node is communicating, it means it is utilizing power. The consumption of battery power by a node indicates that the power is used for computing the routing table and maintaining the routing decisions. $\mathrm{CP}$ can be calculated as:

$$
\begin{aligned}
& \text { PowerConsumptionofnodek }= \\
& \text { TotalPower }-\sum_{k} \text { powerofnodekattimet } \\
& \text { TotalPower }=\sum_{k} \text { energyofnodekattimet }
\end{aligned}
$$

However, it has been studied that packet vs. battery power is used as a metric of network performance. However, when the residual battery power of all the communication nodes is high, the ad-hoc network efficiency will be affected.

The throughput metric represents the average rate of successful data packets delivered from the source node to the destination node through the network communication channel. For evaluation, the ns2.29 simulator tool is used for the implementation of MTPR, EPAR, and DSR. As per simulation results, the EPAR performs better as compared to other routing protocols.

\section{Proposed Cross Layer Design (CLD) for Efficient Power and Routing}

The proposed Cross-layer design communication ensures efficient power and routing among the physical and network layers. Figure 1. depicts the cross layer design (CLD) for efficient power and routing.

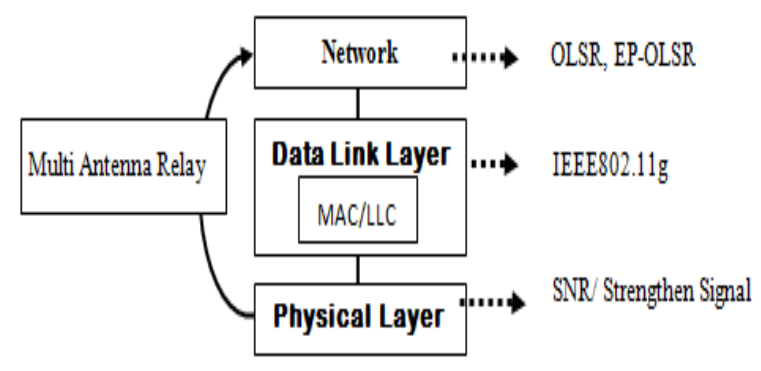

Figure 1. Cross layer design for efficient power and routing
The multi-antenna relay is used to send the strengthened signal to the network layer. The strengthened signal received by the network layer initiates the hello messages to be aware of the connected neighbors and to update the routes by selecting the best MPR. This cross-layer design ensures the link availability, which increases the efficiency of routing due to combining effect of the optimal transmit power in request to send (RTS), clear to send (CTS), and acknowledgments of the link layer for data packet at each hop. This provides the link availability and minimizes the probability of link break among the neighbor UAVs. The efficient power is controlled at the MAC layers, CTS, DATA, and acknowledgment (ACK) information packets are sent at an optimized transmit power level in order to ensure the communication.

The process is followed by the received signal strength (RSS) at the UAV node by integrating the above-discussed packets, which ensure the availability of links.

The proposed design uses the omnidirectional and directional antennas in terms of the multi-antenna relay. In order to ensure the efficient power of signals from the node to Mac and the routing layer interactions, the Mac layer RTS, CTS, DATA, and ACK information packets are transmitted at the lowest power. The packet header field contains RTS, CTS, DATA, and ACK information and includes a field of transmission power level used to manipulate the packet at optimum power. The IEEE $802.11 \mathrm{~g}$ is used to send and transmit RTS, CTS, DATA, and ACK packets, to the UAV node that receives the packet by using the relay.

\subsection{Algorithm for Efficient Power}

Begin

Let Pt $[\mathrm{L}]=3$

Step 1: Read the power table // Check the power table $(\mathrm{Pt})$ at the transmitter node for the destination/ receiver node address and its stored power value Ptt.

Step 2 If not available in the table // If node entry is available, then $\mathrm{Pt}[\mathrm{L}]=\mathrm{Ptt}$

Step 3 RTS power = node entry (power) // Add this power value in the header of RTS and send RTS. 
Step 4 Transmit RTS

Step 5 Extract transmit power (CTS) // extract the power CTS value at node. Calculate the optimized transmit power.

Step 6 Calculate optimum transmit power (DATA)

Step 7 Update (power table) // Update the power table.

Step 8 DATA power $=$ power L $($ power table $) / /$ Add the power level in Data Packet and send Data Packet with updated power value

Step 9 Send DATA packet

Step 10 Read ack

Step 11 end

\subsection{Implementation of EPOLSR Routing Protocol}

The proposed protocol is an extension of the OLSR routing protocol known as efficient power optimized link state routing (EPOLSR) Protocol. This protocol has been implemented and evaluated by developing the testbed experiment scenarios in the UAVCN environment. By using different parameters EPOLSR, OLSR, performance is observed in experiment \#01, and in experiment $\# 02$, the performance of EPOLSR, OLSR, DSR, and AODV protocols is observed.

\section{Research Methodology}

The experimental method is used for the implementation of routing protocols. However, the OPNET modeler research tool has been used for experiment\#01. The OLSR and the proposed EPOLSR routing protocols have been implemented by using cross layer design in the 802.11g environment.

\section{Experiment \#01}

\subsection{Experimental Setup}

The experiment has been carried out by using the simulator tool. The scenario has been developed by using 50 UAV nodes. The routing protocol OLSR has been configured in the scenario with the default setting. The WLAN physical characteristics of standard $802.11 \mathrm{~g}$ are used. The main characteristics of this scenario are shown in Table 1. Similarly, the EPOLSR has been configured by using the same wireless LAN physical characteristics standard $802.11 \mathrm{~g}$ environment and the simulation has been run.

Table 1. Characteristics of the Testbed Scenario Experiment \#01

\begin{tabular}{|l|l|}
\hline \multicolumn{1}{|c|}{ Parameters } & \multicolumn{1}{c|}{ Values } \\
\hline Simulation Environment & OPNET Modeler \\
\hline Routing Protocols & OLSR, EPOLSR \\
\hline Size of Network & $3000 \times 3000 \mathrm{~m}^{2}$ \\
\hline Number of UAVs & 50 \\
\hline DataRate & $54 \mathrm{Mbps}$ \\
\hline Application & Video_Streaming \\
\hline Depiction of_Application & High_Browsing \\
\hline WLAN Phy: Characteristic & IEEE_802.11g \\
\hline Mobility_Model & Random_Waypoint \\
\hline Simulation running time & 800 Sec \\
\hline
\end{tabular}

The 50 UAVs nodes scenario is developed for a network size of $3000 \times 3000 \mathrm{~m}^{2}$. The application, profile, mobility, and nodes are configured. After the application, the attributes of the application are configured as video streaming. Subsequently, the application definition is set at high browsing. Then, the profile definition is set for the FTP and HTTP services. The mobility model random waypoint is used for video streaming. The WLAN physical features and characteristics are configured by using IEEE $802.11 \mathrm{~g}$. Then the chosen data rate set at $54 \mathrm{Mbps}$, the configured channel setting which is auto assigned, the tx power configured by using 0.005 watts, the fragmentation-threshold, which is 1024 and the buffer-size which is 256000 are the parameters used and updated in all the nodes of the OLSR.

A similar configuration is carried out by using EPOLSR and implementing the cross-layer design proposed model. The network simulation running time is set at $800 \mathrm{sec}$, and in the simulation window, the kernel preference is selected as the optimized. Then the simulation is run. With the same configuration parameters, DSR and AODV are configured and simulated. Subsequently, upon completion of the simulation, the results are collected and discussed below. 


\subsection{Results and Discussion}

The results are observed, theoretically explained, and illustrated below.

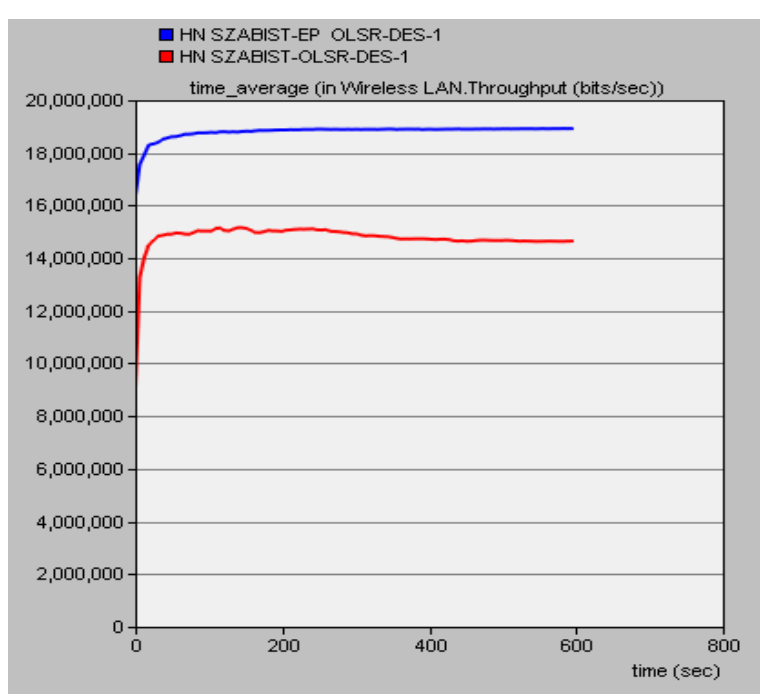

Figure 2. EPOLSR vs. OLSR throughput

The results show that in the 802.11 WLAN environment, the OLSR throughput is $15 \mathrm{Mbps}$, as delineated in Figure 2 above. However, EPOLSR throughput is $18.8 \mathrm{Mbps}$, when 50 nodes are used in the scenario. It has been observed that the EPOLSR throughput is greater as compared to OLSR. In terms of throughput metric the EPOLSR performance better than the OLSR.

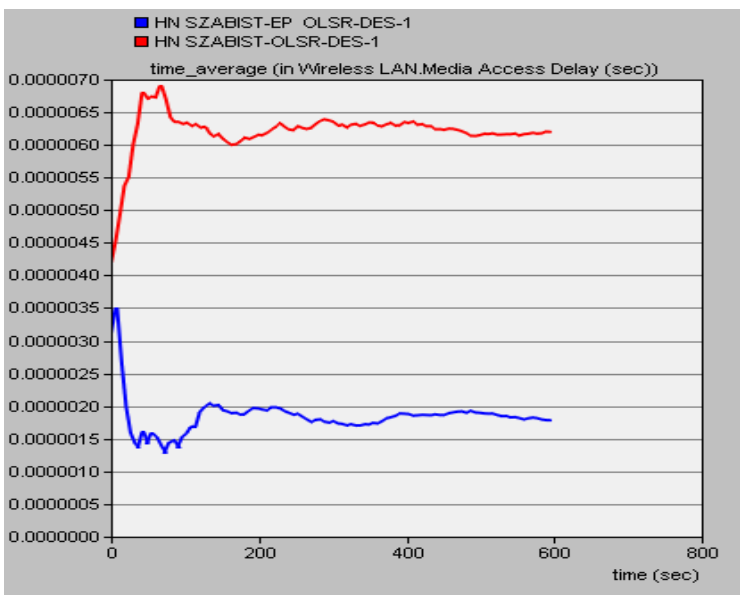

Figure 3. EPOLSR vs. OLSR media access delay

The results show that, in the 802.11 WLAN environment, the OLSR produces a 0.0000063 sec WLAN media access delay, as delineated in Figure 3 above. However, the EPOLSR produces a $0.0000020 \mathrm{sec}$ WLAN media access delay in the scenario of 50 nodes.

\section{Experiment \#02}

\subsection{Experimental Setup}

The experiment has been carried out by using the simulator tool. The scenario has been developed by using 15 nodes. The routing protocol OLSR has been configured in the scenario with the default setting. The WLAN physical characteristics of standard $802.11 \mathrm{~g}$ are used. The main characteristics of this scenario are shown in Table 2. After this experimental setup, the scenario has been accomplished. Similarly, the EPOLSR has been configured by using the same wireless LAN physical characteristics standard cross layer design in the $802.11 \mathrm{~g}$ environment, and the simulation has been run. DSR and AODV are also set in experiment $\# 02$.

Table 2. Characteristics of the Testbed Scenario Experiment \#02

\begin{tabular}{|l|l|}
\hline \multicolumn{1}{|c|}{ Parameters } & \multicolumn{1}{c|}{ Values } \\
\hline Simulation Environment & OPNET Modeler \\
\hline Routing Protocols & $\begin{array}{l}\text { AODV, DSR, OLSR } \\
\text { \& EPOLSR }\end{array}$ \\
\hline Size of Network & $3000 \times 3000 \mathrm{~m}^{2}$ \\
\hline Number of UAVs & 15 \\
\hline Data_Rate & $54 \mathrm{Mbps}$ \\
\hline Application & Video_Streaming \\
\hline Depiction of_Application & High_Browsing \\
\hline WLAN Phy: Characteristic & IEEE_802.11g \\
\hline Mobility Model & Random_Waypoint \\
\hline Simulation Time & 250 Sec \\
\hline
\end{tabular}

The 15 UAVs nodes scenario is developed, having a network size of $3000 \times 3000 \mathrm{~m}^{2}$. The application, profile, mobility, and nodes are configured. After the application, the attributes of the application is updated by selecting the video streaming. Subsequently, the application definition is set at high browsing. Then, the Profile definition is set for the FTP and HTTP services. The mobility model random waypoint is used for video streaming.

The WLAN physical features and characteristics are configured by using IEEE $802.11 \mathrm{~g}$. Then the chosen data rate set at $54 \mathrm{Mbps}$, the configured channel setting which is auto assigned, the tx power configured by using 0.005 watts, the fragmentation-threshold, which is 1024 and the buffer-size which is 256000 are the parameters used and updated in all the UAV nodes of the OLSR. 
A similar configuration is carried out by using EPOLSR and implementing the cross-layer design proposed model. The network simulation running time is set at $250 \mathrm{sec}$, and in the simulation window, the kernel preference is selected as optimized. Then the simulation is run. With the same configuration parameters, DSR and AODV are configured and simulated. Subsequently, upon completion of the simulation, the results are collected and discussed below.

\subsection{Results and Discussion}

The four parameters considered in the experiment are the received routing traffic, the WLAN data dropped, the throughput, and the medium access delay. The results are discussed below and are graphically illustrated in Figures 4, 5, 6, and 7.

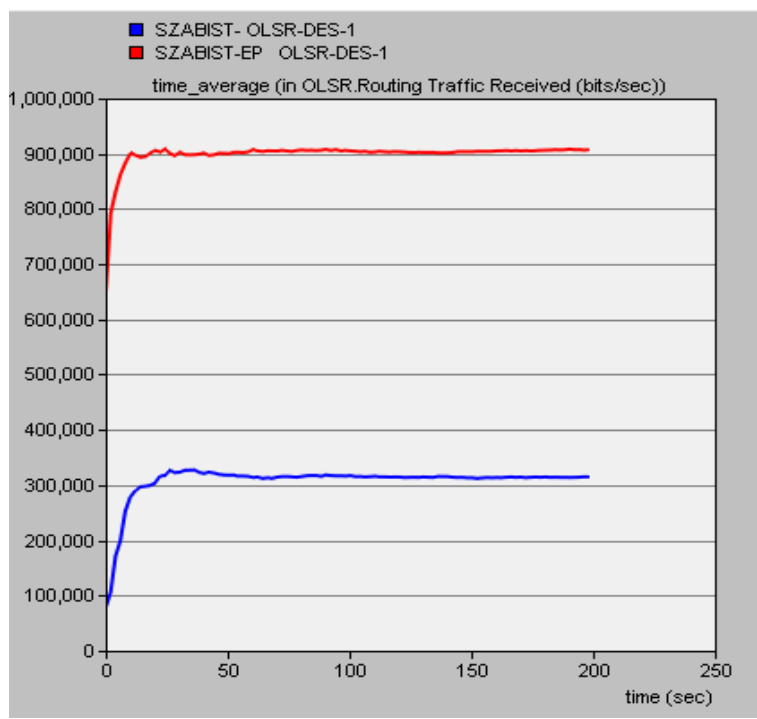

Figure 4. EPOLSR vs. OLSR traffic received

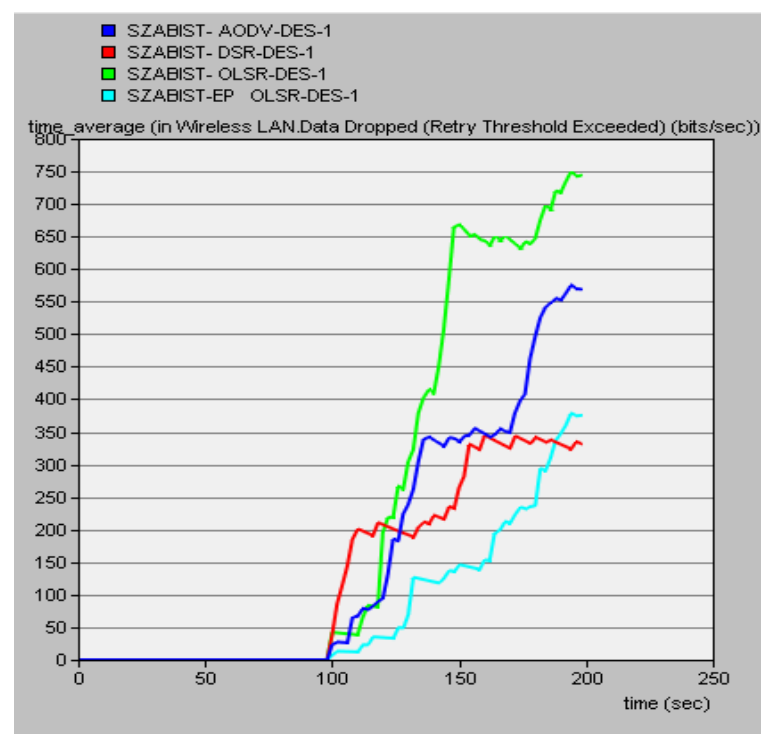

Figure 5. AODV, DSR, OLSR vs. EPOLSR WLAN data dropped

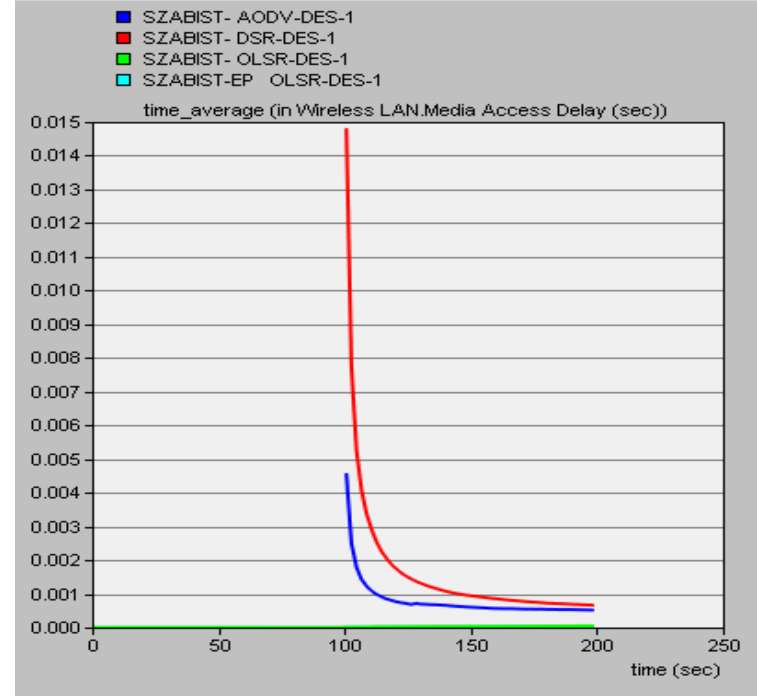

Figure 6. AODV, DSR, OLSR vs. EPOLSR WLAN media access delay

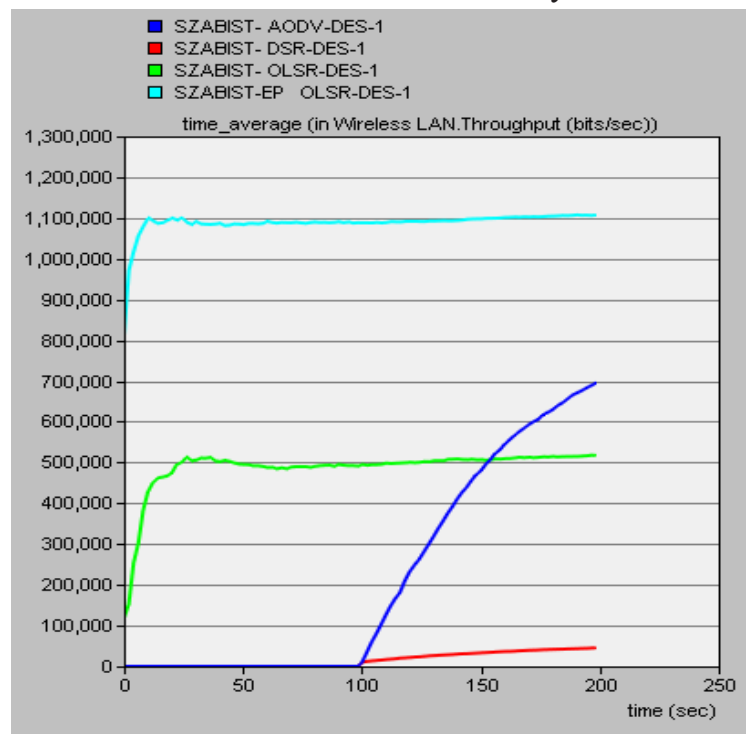

Figure 7. AODV, DSR, OLSR vs. EPOLSR WLAN throughput

The results show that, in the 802.11 WLAN environment, the received OLSR routing traffic is $0.3 \mathrm{Mbps}$, as delineated in Figure 4 above. However, the received EPOLSR routing traffic is $0.9 \mathrm{Mbps}$, in the scenario of 15 nodes. It has been observed that the EPOLSR traffic received is greater as compared to OLSR. In terms of routing traffic receieved metric the EPOLSR performance better than the OLSR.

The results show that, in the 802.11 WLAN environment, the WLAN data decreases 550 bps when AODV is used, the WLAN data decreases 348 bps when DSR is used, the WLAN data dropped 750 bps when OLSR is used, and the WLAN data decreases 352 bps when EPOLSR is used as delineated in Figure 5. It has been observed that in the EPOLSR WLAN data decreses is less as compared to OLSR, DSR and AODV. 
The results show that, in the 802.11g WLAN environment, the AODV produces a $0.0045 \mathrm{sec}$ WLAN media access delay, the DSR produces a $0.015 \mathrm{sec}$ WLAN media access delay, and the OLSR produces a $0.0008 \mathrm{sec}$ WLAN media access delay, as delineated in Figure 6 above. However, the EPOLSR produces a $0.0001 \mathrm{sec}$ WLAN media access delay. It has been observed that the EPOLSR produces less media access delay as compared to OLSR, DSR and AODV.

The results show that, in the 802.11g WLAN environment, the AODV throughput is $0.7 \mathrm{Mbps}$, the observed DSR throughput is $0.05 \mathrm{Mbps}$, the observed OLSR throughput is $0.5 \mathrm{Mbps}$, as delineated in Figure 7 above. However, the EPOLSR throughput is $1.1 \mathrm{Mbps}$. Overall, it has been found that the EPOLSR protocol performs better than OLSR, DSR and AODV protocols.

\section{Conclusion}

This paper explores the routing protocols of the UAVs network and proposes a cross-layer design for efficient power and routing, which is implemented by using the OPNET modeler. The experimental testbed scenarios are developed,

\section{REFERENCES}

Alshabtat, A. I. \& Dong, L. (2011). Low Latency Routing Algorithm for Unmanned Aerial Vehicles Ad-Hoc Networks, International Journal of Electrical, Computer, Energetic, Electronic and Communication Engineering, 5(8), 984-990.

Amin, R., Hussain, M., Ismail, A. \& Iqbal, M. M. (2015). A cross layer design for energy efficiency in peer to peer application over manet, Science International Journal, 28(6), 5183-5187.

Bekmezci, I., Sahingoz, O. K. \& Temel, Ş. (2013). Flying ad-hoc networks (FANETs): A survey, Ad Hoc Networks, 11(3), 1254-1270. DOI: $10.1016 /$ j.adhoc.2012.12.004

Bennis, I., Piamrat, K., Fouchal, H. \& Ayaida, M. (2017). A cross-layer scheme for multimedia transfer over AdHoc networks. In IEEE International Conference on Communications (ICC) (pp. 1-6). DOI: 10.1109/ICC.2017.7997018

Casaquite, R. \& Hwang, W.-J. (2006). Evaluation and Optimization of Joint Scheduling, Power Control, and Routing in Ad Hoc Wireless Networks. In International Conference on and the OLSR and EPOLSR routing protocols are implemented by using the same WLAN features of the IEEE 802.11g in experiment \#01. The first testbed scenario is designed with a 50 nodes density for video streaming application. Nevertheless, the performance of the OLSR and EPOLSR has also been evaluated in this study. By examining the performance of the OLSR and EPOLSR protocols, it has been observed that the proposed routing protocol EPOLSR performs better when compared to OLSR. In the second testbed experiment $\# 02$, the existing conventional protocols OLSR, DSR, and AODV are compared with EPOLSR. However, the efficient power optimized link state routing protocol (EPOLSR) has been found better as it increases the throughput and minimizes the media access delay.

\section{Acknowledgements}

The authors of this article would like to acknowledge the unconditional and continued support of SZABIST, in terms of providing resources and encouraging environment. The research work presented in this paper has not been supported by any organization.

Hybrid Information Technology (pp. 697-704). DOI: 10.1109/ICHIT.2006.133

Chen, L., Zhang, Q., Li, M. \& Jia, W. (2007). Joint Topology Control and Routing in IEEE 802.11-Based Multiradio Multichannel Mesh Networks, IEEE Transactions on Vehicular Technology, 56(5), 3123-3136. DOI:

10.1109/TVT.2007.900509

Conti, M., Maselli, G., Turi, G. \& Giordano, S. (2004). Cross-layering in mobile ad hoc network design, Computer, 37(2), 48-51. DOI: 10.1109/ MC.2004.1266295

Cruz, E. (2018). A comprehensive survey in towards to future FANETs, IEEE Latin America Transactions, 16(3), 876-884. DOI: 10.1109/ TLA.2018.8358668

Divya, M., Subasree, S. \& Sakthivel, N. K. (2015). Performance Analysis of Efficient Energy Routing Protocols in MANET, Procedia Computer Science, 57, 890-897. DOI: 10.1016/j. procs.2015.07.504 
Dolicanin, E., Fetahovic, I., Tuba, E., CaporHrosik, R. \& Tuba, M. (2018). Unmanned combat aerial vehicle path planning by brain storm optimization algorithm, Studies in Informatics and Control, 27(1), 15-24. DOI: 10.24846/ v27i1y201802

Ghada, K., Li, J., Ji, Y. \& Wang, G. (2009). Crosslayer approach for energy efficient routing in WANETs. In IEEE 6th International Conference on Mobile Adhoc and Sensor Systems (pp. 393402). DOI: 10.1109/MOBHOC.2009.5336975

Gu, C. \& Zhu, Q. (2013). A Cross-Layer Routing Protocol for Mobile Ad Hoc Networks Based on Minimum Interference Duration, Applied Mechanics and Materials, 347-350, 2028-2032.

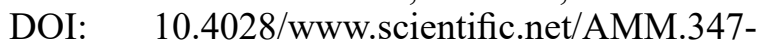
350.2028

Gupta, L., Jain, R. \& Vaszkun, G. (2016). Survey of important issues in UAV communication networks, IEEE Communications Surveys \& Tutorials, 18(2), 1123-1152. DOI: 10.1109/ COMST.2015.2495297

Jonson, T., Pezeshki, J., Chao, V., Smith, K. \& Fazio, J. (2008). November. Application of delay tolerant networking (DTN) in airborne networks. In MILCOM 2008-2008 IEEE Military Communications Conference (pp.1-7). DOI: 10.1109/MILCOM.2008.4753464

Kawadia, V. \& Kumar, P. R. (2005). Principles and protocols for power control in wireless ad hoc networks, IEEE Journal on Selected Areas in communications, 23(1), 76-88. DOI: 10.1109/ JSAC.2004.837354

Khan, Z. A., Awais, M., Alghamdi, T. A., Khalid, A., Fatima, A., Akbar, M. \& Javaid, N. (2019). Region Aware Proactive Routing Approaches Exploiting Energy Efficient Paths for Void Hole Avoidance in Underwater WSNs, IEEE Access, 7, 140703-140722. DOI: 10.1109/ ACCESS.2019.2939155

Li, D., Jia, X. \& Du, H. (2006). QoS Topology Control for Nonhomogenous Ad Hoc Wireless Networks, EURASIP Journal on Wireless Communications and Networking, 2006(1), 1-10. DOI: $10.1155 / \mathrm{WCN} / 2006 / 82417$

Manyam, S. G., Sundar, K. \& Casbeer, D. W. (2020). Cooperative Routing for an Air-Ground Vehicle Team--Exact Algorithm, Transformation Method, and Heuristics, IEEE Transactions on Automation Science and Engineering, 17(1), 537-547. DOI: 10.1109/TASE.2019.2931894
Rajan, S., Sundar, K. \& Gautam, N. (2019). Routing Problems for Reconnaissance Patrolling Missions. In International Conference on Unmanned Aircraft Systems (ICUAS) (pp. 213-220).

Rosati, S., Krużelecki, K., Traynard, L. \& Mobile, B. R. (2013). Speed-aware routing for UAV adhoc networks. In IEEE Globecom Workshops (GC Wkshps) (pp. 1367-1373). DOI: 10.1109/ GLOCOMW.2013.6825185

Shakhatreh, H., Sawalmeh, A. H., Al-Fuqaha, A., Dou, Z., Almaita, E., Khalil, I., Othman, N. S., Khreishah, A. \& Guizani, M. (2019). Unmanned aerial vehicles (UAVs): A survey on civil applications and key research challenges, IEEE Access, 7, 48572-48634. DOI: 10.1109/ ACCESS.2019.2909530

Shirani, R., St-Hilaire, M. \& Kunz, T. (2012). A Cross-Layered Communication Architecture for Search and Surveillance Applications of Unmanned Aeronautical AdHoc Networks. In 8th International Conference on Wireless Communications, Networking and Mobile Computing (pp 1-5). DOI: 10.1109/ WiCOM.2012.6478274

Singh, K. \& Verma, A. K. (2014). Applying OLSR routing in FANETs. In IEEE International Conference on Advanced Communications, Control and Computing Technologies (pp. 12121215). DOI: 10.1109/ICACCCT.2014.7019290

Srinivasan, S., Latchman, H., Shea, J., Wong, T. \& McNair, J. (2004). Airborne Traffic Surveillance Systems: Video Surveillance of Highway Traffic. In Proceedings of the ACM 2Nd International Workshop on Video Surveillance \& Sensor Networks (pp. 131-135). DOI: $10.1145 / 1026799.1026821$

Wang, Z., Chen, Y. \& Li, C. (2013). PSR: A lightweight proactive source routing protocol for mobile ad hoc networks, IEEE transactions on Vehicular Technology, 63(2), 859-868. DOI: 10.1109/TVT.2013.2279111

Zeng, Y., Xu, J. \& Zhang, R. (2019). Energy minimization for wireless communication with rotary-wing UAV, IEEE Transactions on Wireless Communications, 18(4), 2329-2345. DOI: 10.1109/TWC.2019.2902559

Zheng, Y., Wang, Y., Li, Z., Dong, L., Jiang, Y. \& Zhang, H. (2014). A Mobility and Load aware OLSR routing protocol for UAV mobile adhoc networks. In International Conference on Information and Communications Technologies (pp. 1-7). DOI: 10.1049/cp.2014.0575 